\title{
Comparative evaluation of network features for the prediction of breast cancer metastasis
}

\author{
Nahim Adnan ${ }^{1}$, Zhijie Liu², Tim H.M. Huang ${ }^{2}$ and Jianhua Ruan ${ }^{1,2 *}$ \\ From The International Conference on Intelligent Biology and Medicine (ICIBM) 2019 \\ Columbus, OH, USA. 9-11 June 2019
}

\begin{abstract}
Background: Discovering a highly accurate and robust gene signature for the prediction of breast cancer metastasis from gene expression profiling of primary tumors is one of the most challenging tasks to reduce the number of deaths in women. Due to the limited success of gene-based features in achieving satisfactory prediction accuracy, many methodologies have been proposed in recent years to develop network-based features by integrating network information with gene expression. However, evaluation results are inconsistent to confirm the effectiveness of network-based features, because of many confounding factors involved in classification model learning process, such as data normalization, dimension reduction, and feature selection. An unbiased comparative evaluation is essential for uncovering the strength of network-based features.
\end{abstract}

Methods: In this study, we compared several types of network-based features obtained using different mathematical operators (Mean, Maximum, Minimum, Median, Variance) on geneset (i.e., a gene and its' neighbors in the network) in protein-protein interaction network and gene co-expression network for their ability in predicting breast cancer metastasis using gene expression data from more than 10 patient cohorts.

Results: While network-based features are usually statistically more significant than gene-based feature, a consistent improvement of prediction performance using network-based features requires a substantial number of patients in the dataset. In contrary to many previous reports, no evidence was found to support the robustness of network-based features and we argue some of the robustness may be due to the inherent bias associated with node degree in the network. In addition, different types of network features seem to cover different pathways and are complementary to each other. Consequently, an ensemble classifier combining different network features was proposed and was found to significantly outperform classifiers based on gene-based feature or any single type of network-based features.

Conclusions: Network-based features and their combination show promise for improving the prediction of breast cancer metastasis but may require a large amount of training data. Robustness claim of network-based features needs to be re-examined with network node degree and other confounding factors in consideration.

Keywords: Breast cancer metastasis, Metastasis prediction, Network features, Gene expression analysis

\footnotetext{
*Correspondence: jianhua.ruan@utsa.edu

${ }^{1}$ Department of Computer Science, University of Texas at San Antonio, One

UTSA Circle, San Antonio, TX 78249, USA

${ }^{2}$ Department of Molecular Medicine, University of Texas Health Science Center

at San Antonio, 7703 Floyd Curl Drive, San Antonio, TX 78230, USA
}

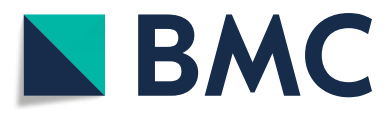

(C) The Author(s). 2020 Open Access This article is distributed under the terms of the Creative Commons Attribution 4.0 International License (http://creativecommons.org/licenses/by/4.0/), which permits unrestricted use, distribution, and reproduction in any medium, provided you give appropriate credit to the original author(s) and the source, provide a link to the Creative Commons license, and indicate if changes were made. The Creative Commons Public Domain Dedication waiver (http://creativecommons.org/publicdomain/zero/1.0/) applies to the data made available in this article, unless otherwise stated. 


\section{Background}

The most frequently diagnosed disease and the second leading cause of death in western women have been identified as breast cancer [1]. According to the American Cancer Society [2], US women have $12.4 \%$ (about 1 in 8) chance of developing breast cancer over the course of their lifetime. About 5\% of women have metastatic (i.e., recurrence of cancer) breast cancer at their first diagnosis [3]. Only lymph node status, histology and tumor size of the patients are not sufficient to determine breast cancer metastasis [4]. Due to the availability of gene expression data for primary cancerous tumors, many methods have been developed to predict breast cancer metastasis outcomes over the last decade. The patient being free from recurrence for at least 5 years and relapse occurring within 5 years after the first diagnosis are termed as good and poor outcomes respectively.

Initially, single gene-based prognostic signatures that are highly differential between good and poor outcomes were proposed [1, 5-7]. Inconsistencies of prognostic signatures were discovered when single gene-based prognostic signatures varied in different studies [8]. There was no homogeneity within the found signatures which complicated the biological relevance of the metastatic outcomes. A lot of genes are correlated with the metastatic outcome which makes it possible to identify multiple single genebased signatures from the same dataset $[8,9]$. Another problem in the gene expression analysis is that the data available is very high dimensional, for example- the number of genes is 10,000 for only hundreds of patients. Many studies tried to pool multiple datasets together to lessen the $\mathrm{n}>>\mathrm{p}$ problem of the expression data and it provided higher statistical significance of the results [9-11]. Many samples are needed to identify robust gene signature and to overcome the issues of dataset heterogeneity. The number of samples needed to achieve an improvement on classification accuracy is still unexplored and more studies are required to accurately address this concern.

From a biological standpoint, protein works in complexes and the aberrant nature of these complexes can cause cancer. Motivated by this assumption, many methodologies have been proposed integrating network information such as- protein-protein interaction network, coexpression network and cellular pathway information with the gene expression data for better classification accuracy and robust gene signature identification [12-22]. By integrating network with gene expression, those methods tried to find protein complexes or genesets which better distinguished between the good and poor outcome of the metastasis. The aggregated expression of the genes belonging to a geneset was used as the network-based feature in most of the methods. An initial feature selection step was applied to refine the network-based features before creating the final classification model in some of the previously proposed methods $[12,16]$.

Although many studies claim improved classification accuracy and gene signature stability, there has been insufficient comparative analysis between network-based methods and single gene-based approaches. Two recent studies [23, 24] compared single gene-based approach with the network-based ones and reported that networkbased methods do not perform better over the single genebased approach in terms of classification accuracy and the signature stability. Later, in [21], the authors pointed out that selecting network-based features based on statistical significance hinders the classification performance of the network-based methods. They also argued that only using the average operator to create network-based features does not improve classification accuracy significantly. Other types of operator (i.e., maximum, minimum, median, variance) should also be used in the models.

The current study mainly focused on the differential analysis of the different feature types by comparing network-based features with the single gene-based feature. Amsterdam Classification Evaluation Suite (ACES) [24], a compilation of gene expressions of twelve (12) separate studies, was used for the analysis. The goal of the study was to determine the number of significant features that passed the significance threshold value for separate studies. The impact of the number of samples, classification accuracy of feature types and robustness of gene signature across studies were thoroughly examined. An ensemble classifier CNF (Combining multiple Network-based Features) was proposed which provides improved performance over individual network-based features. Moreover, Gene Ontology analysis was done to find the biological interpretation of the top significant genes from separate studies.

\section{Methods}

\section{Gene expression data}

The gene expression dataset used in this analysis is ACES [24] dataset. It combined breast cancer patient samples from 12 different studies together from NCBIs Gene Expression Omnibus. The dataset took the 133A platform into account and removed duplicate samples with the same GEO id in multiple studies. Sample array quality control checking was done, and outlier samples were discarded after RLE (Relative Log Expression) or NUSE (Normalized Unscaled Standard Error Plot) analysis. This yielded a cohort of 1616 patients from 12 studies and alltogether the patient expression arrays were normalized using justRMA method from $R$. Probe intensities of samples were log-normalized and mean centered. Finally, after discarding missing, null or zero values there were 12,750 gene probes in ACES dataset [24] . The class label for each patient was determined as good or poor outcome based on 
the recurrence free survival time where the threshold was set to 5 years. The detailed information about the studies and the class distributions are provided in the Table 1.

\section{Protein-protein interaction network}

The protein-protein interaction network (PPI) was created from the BioGrid (version 3.4.149) interaction database [25]. The interaction network only contained the genes from the ACES dataset where self-edges were discarded which finally produced 180,371 edges for 12,750 nodes (no. of genes).

\section{Gene co-expression network}

A global co-expression network was created for the analysis based on mutual k-nearest neighbors of genes using Pearson correlation coefficients between the genes' expression in the ACES dataset (i.e., two genes were connected if they were within the top- $k$ most co-expressed genes from each other.) The number of neighbors $(k)$ was set to 84 so that the network can have a similar number of edges as in the PPI network. This yielded a network containing 161,042 edges for 12,750 nodes (no. of genes) and the degree distribution approximately followed a power-law distribution like the PPI network.

\section{Feature types}

Each gene and its' neighbors from the network were considered as a geneset. Multiple network-based features were evaluated using different mathematical operators (MAX, MIN, MEDIAN, MEAN, VARIANCE) on the geneset. The specification of the feature types is given in Table 2, where abbreviation "CE" stands for CoExpression network and "PPI" for Protein-Protein Interaction network. CEEdge and PPIEdge are the two feature types that consider each of the edges of network as features.

Table 1 Specification of the studies in ACES

\begin{tabular}{lllll}
\hline Dataset & Geo accession no. & No. of poor & No. of good & Total patient \\
\hline Desmedt & 7390 & 56 & 127 & 183 \\
Hatzis & 25066 & 102 & 48 & 150 \\
Ivshina & 4922 & 30 & 72 & 102 \\
Loi & 6532 & 24 & 33 & 57 \\
Pawitan & 1456 & 33 & 114 & 147 \\
Miller & 3494 & 21 & 68 & 89 \\
Minn & 2603 & 21 & 44 & 65 \\
Schmidt & 11121 & 24 & 145 & 169 \\
Symmans & 17705 & 37 & 187 & 224 \\
WangY & 5327 & 10 & 42 & 52 \\
WangYE & 2034 & 88 & 169 & 257 \\
Zhang & 12093 & 9 & 112 & 121 \\
ACES & & 455 & 1161 & 1616 \\
\hline
\end{tabular}

Table 2 Specification of the feature types

\begin{tabular}{|c|c|}
\hline Name & Details \\
\hline Gene & $\begin{array}{l}\text { Using gene expression without integrating any network } \\
\text { information. }\end{array}$ \\
\hline CENO & $\begin{array}{l}\text { A genes' expression is based on the average expression of } \\
\text { its neighbors only. }\end{array}$ \\
\hline CEMEAN & The mean of the expression of a gene and its neighbors. \\
\hline CEMAX & The maximum of the expression of a gene and its neighbors. \\
\hline CEMIN & The minimum of the expression of a gene and its neighbors. \\
\hline CEMED & The median of the expression of a gene and its neighbors. \\
\hline CEVAR & The variance of the expression of a gene and its neighbors. \\
\hline CEEdge & $\begin{array}{l}\text { Each edge is the summation of the expression of its } \\
\text { corresponding genes. }\end{array}$ \\
\hline PPINO & $\begin{array}{l}\text { A genes' expression is based on the average expression of } \\
\text { its neighbors only. }\end{array}$ \\
\hline PPIMEAN & The mean of the expression of a gene and its neighbors. \\
\hline PPIMAX & The maximum of the expression of a gene and its neighbors. \\
\hline PPIMIN & The minimum of the expression of a gene and its neighbors. \\
\hline PPIMED & The median of the expression of a gene and its neighbors. \\
\hline PPIVAR & The variance of the expression of a gene and its neighbors. \\
\hline PPIEdge & $\begin{array}{l}\text { Each edge is the summation of the expression of its } \\
\text { corresponding genes. }\end{array}$ \\
\hline
\end{tabular}

\section{Identification of significant features}

First, for each feature, its value is calculated for each patient depending on the feature types (see Table 2). Student's t-test is then used to compare feature values between the good and poor outcome patients in a dataset, and a $p$-value is computed for each feature. Then, we computed False Discovery Rate (FDR) based on Benjamini and Hochberg method [26] and an FDR corrected $p$ value threshold was set to 0.1 for selection of significant features.

\section{Robustness measure of features}

To evaluate the robustness for different feature types across different studies, we selected the top-160 most statistically significant features (ranked by Student's ttest $p$-value) from each gene- or network-based features (except CEEdge and PPIEdge). The number 160 was chosen so that the expected overlap between the features from two different datasets is $160 \times 160 / 12750=2$ (160 genes chosen from 12,750 genes). For CEEdge and PPIEdge, we pooled the genes associated with the topranked edges so that a total of 160 genes were obtained. Then the actual overlap of genes between each pair of datasets was counted, and the geometric mean of the fold change (observed overlap / expected overlap) across the 66 pairs of datasets was calculated as a measure of the feature's robustness. 


\section{Classification model and performance evaluation}

Logistic regression was used as the classification model for evaluating the prediction performance of different feature types. The area under the receiver-operating characteristics curve (AUC) was used as the performance measurement of the classification model due to the class imbalance nature of the data. For evaluation purpose, average AUC of 10 repetitions of 5 -fold cross-validation was measured for each feature type in each dataset.

\section{Classifier with combined network features (CNF)}

Based on the classification performance and mutual complementariness of individual network-based features, an ensemble classifier based on combined network-based features, CNF, was proposed. To acquire better confidence in classification, CNF utilizes multiple network-based features together, including MEAN, MAX, MIN, MEDIAN features for both co-expression network and PPI network. A logistic regression model is created for each of the eight network-based feature types. When a test instance is provided, CNF obtains predicted probabilities from the eight logistic regression models for that test instance. The final prediction for that particular test instance is done by averaging the predicted probabilities of those individual classifiers.

\section{Results and discussion}

\section{Number of significant features}

Figure 1 shows the number of features passing the FDR corrected $p$-value threshold for each feature type in the 12 cohorts, as well as in the combined ACES dataset. First, almost none of the features were able to pass the threshold in "Desmedt", "Ivshina", "Loi", "Miller", "Minn", "WangY", "Zhang" datasets. This indicated that the number of patients and the class distribution have an impact on feature significance. From Fig. 2 it can be observed that "Ivshina", "Loi", "Miller", "Minn", "WangY", "Zhang" have the lowest number of patients and very imbalanced class distribution. Although the "Desmedt" dataset has relatively more patients and the class distribution is less skewed, no features passed the FDR corrected $p$-value threshold, indicating that the differential analysis also depends on the nature of the dataset. Note that the total number of CEEdge and PPIEdge features are much larger than other network-based features, hence these feature types have the highest number of significant features.

The result of the ACES dataset seems much more stable for each feature type compared to the results of the separate studies. The number of gene features passing the predefined threshold is much higher than separate studies (Fig. 1). Uniform significant number of features for $A C E S$ dataset indicated that a large number of samples is required for attaining the consistent result. The number of significant features for most network-based feature types (except CENO and PPINO) was much higher than the gene-based feature in $A C E S$ dataset, suggesting that network neighbors can provide additional discriminating information that would otherwise be too noisy to identify for individual genes. It is also worth noting that network features resulted from multiple gene-expression values, such as MEAN and VAR, are more abundant than features resulted from operators that only pick one gene (e.g., MIN, MAX, and MEDIAN), as the later can be more affected by noise in the network structure.

\section{Prediction performance of different feature types}

The second analysis was focused on whether the integration of the network with genes offers better classification accuracy compared to gene-based feature (Fig. 3). Out of the 12 independent datasets, gene-based feature

\begin{tabular}{|c|c|c|c|c|c|c|c|c|c|c|c|c|c|}
\hline & Desmedt & Hatzis & Ivshina & Loi & Pawitan & Miller & Minn & Schmidt & Symmans & WangY & WangYE & Zhang & ACES \\
\hline Gene & 0 & 1850 & 0 & \begin{tabular}{|l|l|}
0 & \\
\end{tabular} & 50 & 0 & 0 & 42 & 48 & \begin{tabular}{|l|}
0 \\
\end{tabular} & 69 & 0 & 3374 \\
\hline CENO & 0 & 123 & 0 & 0 & 923 & 0 & 0 & 0 & 0 & 0 & 0 & 0 & 479 \\
\hline CEMEAN & 0 & 1 & 0 & 0 & 1765 & 0 & 0 & 0 & 5 & 0 & 490 & 0 & 6009 \\
\hline CEMAX & 1 & 1806 & 0 & 0 & 9 & 0 & 0 & 1194 & 1530 & 0 & 1080 & 0 & 5490 \\
\hline CEMIN & 0 & 2891 & 0 & 0 & 194 & 0 & 0 & 0 & 1359 & 0 & 151 & 0 & 5201 \\
\hline CEMED & 0 & 3854 & 0 & 0 & 748 & 0 & 0 & 235 & 553 & 0 & 57 & 0 & 4814 \\
\hline CEVAR & 0 & 102 & 0 & 0 & 0 & 0 & 0 & 1712 & 1891 & 0 & 4 & 82 & 6295 \\
\hline CEEdge & 0 & 46050 & 0 & 0 & 6711 & 0 & 0 & 4033 & 5573 & 0 & 1185 & 0 & 63238 \\
\hline PPINO & 0 & 267 & 0 & 0 & 584 & 0 & 0 & 0 & 0 & 0 & 0 & 0 & 750 \\
\hline PPIMEAN & 0 & 0 & 0 & 0 & 384 & 0 & 0 & 0 & 1 & 0 & 0 & 0 & 7135 \\
\hline PPIMAX & 0 & 314 & 0 & 0 & 3 & 0 & 0 & 1957 & 1706 & 0 & 19 & 0 & 5304 \\
\hline PPIMIN & 0 & 404 & 0 & 0 & 9 & 0 & 0 & 1 & 0 & 0 & 2 & 0 & 5338 \\
\hline PPIMED & 0 & 1317 & 0 & 0 & 89 & 0 & 0 & 23 & 67 & 0 & 110 & 0 & 3992 \\
\hline PPIVAR & 0 & 112 & 0 & 0 & 0 & 0 & 0 & 3216 & 977 & 1 & 0 & 64 & 7522 \\
\hline PPIEdge & 0 & 26924 & 0 & 0 & 1723 & 0 & 0 & 360 & 1812 & 0 & 1149 & 0 & 71680 \\
\hline
\end{tabular}

Fig. 1 Number of significant features for each feature type in 13 datasets 


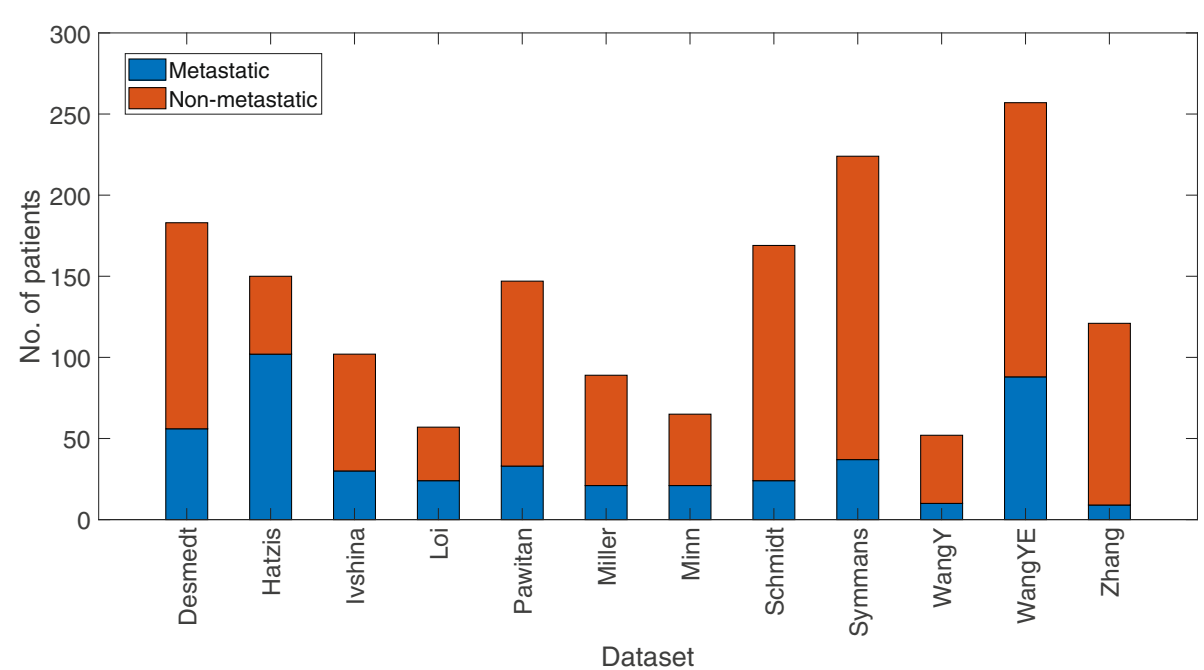

Fig. 2 Patient class distribution in 12 studies

and network-based features had the highest AUC scores on 3 and 9 datasets respectively. For example, PPIMAX is the highest in "Desmedt" dataset whereas CEMIN is the highest in "Ivshina" dataset. Different feature types achieved the highest AUC score in different datasets among the network-based feature types which indicated that the classification accuracy varied across the datasets. No feature type showed consistent improvement across datasets. Although network-based features outperformed gene-based feature in 9 datasets, it is insufficient to claim the effectiveness of network-based over gene-based features among the different studies, given that multiple types of network-based features were tested against the gene-based feature.
However, when tested on the combined ACES dataset, which is relatively free from class imbalance problem and had the highest patient cohort diversity, the network features based on MEAN, MIN, MAX, and MEDIAN operators of gene and its neighbors from both co-expression network and protein-protein interaction network significantly outperformed gene-based feature (Fig. 4). VARIANCE-based features from both PPI and co-expression networks also provided slightly better AUC than the gene-based feature. While CEEdge and PPIEdge features performed on par with the gene-based feature, it is worth noting that the number of features in these feature types is much larger than the other types of features, which could have impeded their classification

\begin{tabular}{|c|c|c|c|c|c|c|c|c|c|c|c|c|c|}
\hline & Desmedt & Hatzis & Ivshina & Loi & Pawitan & Miller & Minn & Schmidt & Symmans & WangY & WangYE & Zhang & ACES \\
\hline Gene & 0.615 & $\underline{0.858}$ & 0.575 & 0.561 & 0.694 & 0.551 & 0.678 & 0.746 & 0.725 & 0.697 & $\underline{0.730}$ & $\underline{0.746}$ & 0.619 \\
\hline CENO & 0.535 & 0.835 & 0.570 & 0.624 & 0.716 & 0.552 & 0.633 & 0.633 & 0.659 & 0.435 & 0.676 & 0.742 & 0.565 \\
\hline CEMEAN & 0.536 & 0.827 & 0.625 & 0.487 & 0.678 & 0.515 & 0.618 & 0.684 & 0.671 & 0.459 & 0.707 & 0.503 & 0.704 \\
\hline CEMAX & 0.602 & 0.812 & 0.561 & 0.568 & 0.602 & 0.521 & $\underline{0.704}$ & 0.740 & 0.710 & 0.685 & 0.701 & 0.616 & 0.682 \\
\hline CEMIN & 0.566 & 0.835 & $\underline{0.675}$ & 0.472 & 0.719 & 0.556 & 0.594 & 0.682 & 0.712 & 0.675 & 0.693 & 0.648 & 0.713 \\
\hline CEMED & 0.605 & 0.848 & 0.524 & 0.485 & 0.680 & 0.527 & 0.647 & $\underline{0.762}$ & 0.733 & 0.657 & 0.713 & 0.707 & 0.687 \\
\hline CEVAR & 0.571 & 0.781 & 0.555 & 0.465 & 0.650 & 0.420 & 0.654 & 0.688 & $\underline{0.761}$ & 0.579 & 0.614 & 0.553 & 0.684 \\
\hline CEEdge & 0.629 & 0.841 & 0.558 & 0.500 & 0.675 & 0.553 & 0.668 & 0.743 & 0.722 & 0.705 & 0.728 & 0.729 & 0.614 \\
\hline PPINO & 0.533 & 0.817 & 0.623 & $\underline{0.735}$ & 0.682 & $\underline{0.587}$ & 0.568 & 0.645 & 0.666 & 0.614 & 0.652 & 0.641 & 0.597 \\
\hline PPIMEAN & 0.584 & 0.795 & 0.565 & 0.501 & 0.671 & 0.556 & 0.619 & 0.629 & 0.655 & 0.520 & 0.689 & 0.645 & $\underline{0.723}$ \\
\hline PPIMAX & $\underline{0.659}$ & 0.800 & 0.605 & 0.497 & 0.658 & 0.525 & 0.619 & 0.748 & 0.697 & 0.589 & 0.645 & 0.607 & 0.680 \\
\hline PPIMIN & 0.494 & 0.824 & 0.603 & 0.406 & $\underline{0.735}$ & 0.520 & 0.644 & 0.603 & 0.618 & $\underline{0.765}$ & 0.672 & 0.589 & 0.669 \\
\hline PPIMED & 0.613 & $\underline{0.858}$ & 0.591 & 0.561 & 0.656 & 0.585 & 0.692 & 0.760 & 0.705 & 0.699 & 0.695 & 0.643 & 0.689 \\
\hline PPIVAR & 0.583 & 0.794 & 0.600 & 0.397 & 0.643 & 0.484 & 0.632 & 0.665 & 0.728 & 0.591 & 0.639 & 0.447 & 0.665 \\
\hline PPIEdge & 0.638 & 0.837 & 0.581 & 0.517 & 0.656 & 0.565 & 0.691 & 0.745 & 0.701 & 0.647 & 0.715 & 0.710 & 0.608 \\
\hline
\end{tabular}

Fig. 3 Classification accuracy of each feature type in 13 datasets. The highlighted entry as "bold" and "underlined" in each dataset indicates that it has the highest average AUC score for that dataset 


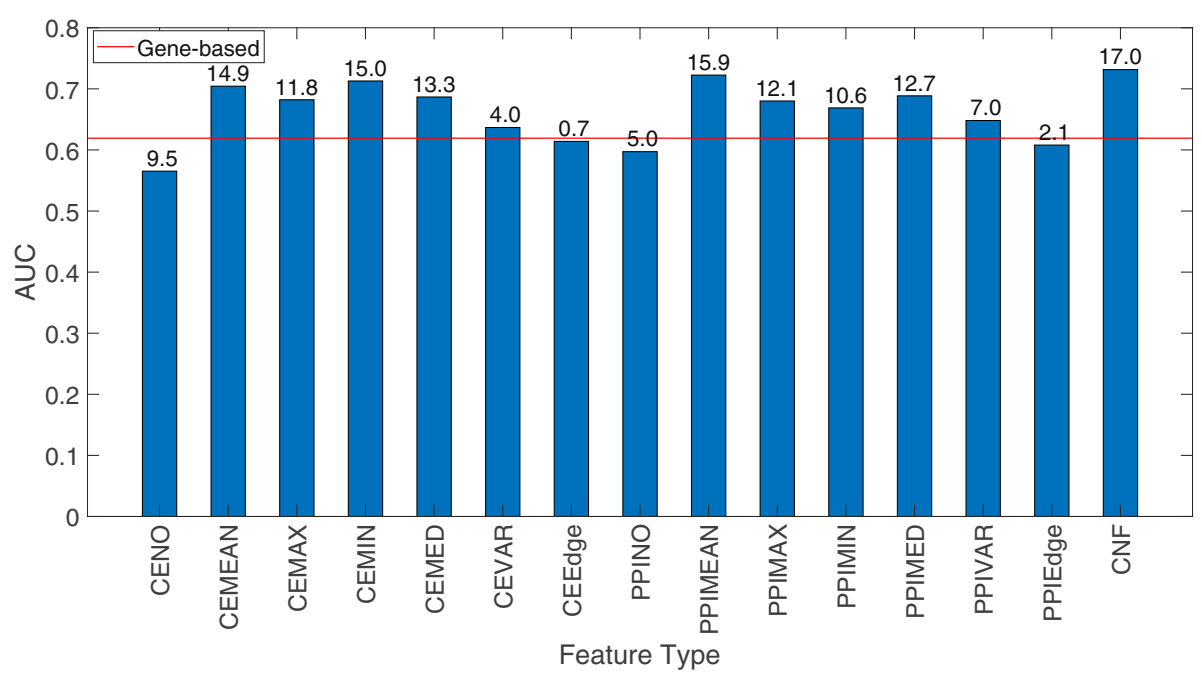

Fig. 4 Comparison of classification accuracy of different network-based features with the gene-based feature for ACES dataset. The red line indicates the average AUC score of gene-based feature. The bars indicate the average AUC score of 10 repetitions for 5-fold cross-validation. The value on top of the bar indicates the -log10(p-value) of the two-sided paired t-test of the AUC scores of the cross-validation folds of indicated feature type with the gene-based feature

accuracy. More investigation may be needed to understand the ways of finding a subset of edges that will offer improved classification accuracy. Finally, Neighbor-Only features (CENO, PPINO) do not seem to have any advantage over gene-based features in improving classification performance. This is also consistent with the results that very few Neighbor-Only features are statistically significant between good and poor outcome groups (Fig. 1).

Overall, using ACES dataset, we showed that integration with PPI or co-expression networks resulted in not only larger numbers of significant features than using gene expression data alone, but also more accurate classifica-

\begin{tabular}{|r|c|c|c|c|c|c|c|c|c|}
\hline & $90 \%$ & $80 \%$ & $70 \%$ & $60 \%$ & $50 \%$ & $40 \%$ & $30 \%$ & $20 \%$ & $10 \%$ \\
\hline Gene & 0.641 & 0.635 & 0.655 & 0.622 & 0.671 & 0.673 & 0.692 & 0.669 & 0.653 \\
CENO & 0.578 & 0.570 & 0.571 & 0.559 & 0.593 & 0.587 & 0.630 & 0.617 & 0.633 \\
CEMEAN & $\mathbf{0 . 7 1 0}$ & $\mathbf{0 . 6 9 9}$ & $\mathbf{0 . 7 0 6}$ & $\mathbf{0 . 6 7 3}$ & $\mathbf{0 . 7 0 6}$ & $\mathbf{0 . 6 9 1}$ & $\mathbf{0 . 7 0 7}$ & 0.627 & 0.652 \\
CEMAX & $\mathbf{0 . 6 8 5}$ & $\mathbf{0 . 6 7 0}$ & $\mathbf{0 . 6 6 2}$ & $\mathbf{0 . 6 6 7}$ & 0.659 & $\mathbf{0 . 6 9 1}$ & $\mathbf{0 . 7 0 3}$ & $\mathbf{0 . 6 9 4}$ & 0.627 \\
CEMIN & $\mathbf{0 . 7 1 7}$ & $\mathbf{0 . 7 0 3}$ & $\mathbf{0 . 7 1 5}$ & $\mathbf{0 . 6 8 1}$ & $\underline{\mathbf{0 . 7 1 6}}$ & $\mathbf{0 . 6 8 2}$ & $\underline{\mathbf{0 . 7 1 3}}$ & 0.669 & 0.644 \\
CEMED & $\mathbf{0 . 6 8 9}$ & $\mathbf{0 . 6 8 9}$ & $\mathbf{0 . 6 9 1}$ & $\mathbf{0 . 6 4 2}$ & $\mathbf{0 . 7 0 1}$ & 0.670 & 0.672 & 0.637 & 0.626 \\
CEVAR & $\mathbf{0 . 6 5 5}$ & $\mathbf{0 . 6 5 3}$ & 0.655 & $\mathbf{0 . 6 5 6}$ & $\mathbf{0 . 6 7 2}$ & 0.650 & 0.566 & 0.655 & 0.600 \\
CEEdge & 0.629 & 0.625 & $\mathbf{0 . 6 6 4}$ & $\mathbf{0 . 6 2 3}$ & 0.667 & 0.662 & 0.677 & 0.641 & 0.615 \\
PPINO & 0.613 & 0.587 & 0.612 & 0.592 & 0.612 & 0.623 & 0.639 & 0.622 & 0.563 \\
PPIMEAN & $\mathbf{0 . 7 2 7}$ & $\mathbf{0 . 7 1 2}$ & $\mathbf{0 . 7 2 2}$ & $\mathbf{0 . 7 0 3}$ & $\mathbf{0 . 7 1 2}$ & $\mathbf{0 . 7 0 2}$ & $\mathbf{0 . 7 0 3}$ & 0.645 & 0.631 \\
PPIMAX & $\mathbf{0 . 6 8 3}$ & $\mathbf{0 . 6 9 0}$ & $\mathbf{0 . 6 7 8}$ & $\mathbf{0 . 6 4 5}$ & $\mathbf{0 . 6 7 2}$ & $\mathbf{0 . 7 0 4}$ & 0.666 & $\mathbf{0 . 6 7 5}$ & 0.637 \\
PPIMIN & $\mathbf{0 . 6 7 8}$ & $\mathbf{0 . 6 6 2}$ & $\mathbf{0 . 6 6 9}$ & $\mathbf{0 . 6 4 8}$ & $\mathbf{0 . 7 1 6}$ & 0.637 & 0.688 & 0.654 & 0.588 \\
PPIMED & $\mathbf{0 . 6 8 5}$ & $\mathbf{0 . 6 8 7}$ & $\mathbf{0 . 6 9 8}$ & $\mathbf{0 . 6 7 5}$ & $\mathbf{0 . 6 9 7}$ & $\mathbf{0 . 6 8 7}$ & 0.674 & 0.662 & $\mathbf{0 . 6 6 0}$ \\
PPIVAR & $\mathbf{0 . 6 6 5}$ & $\mathbf{0 . 6 4 6}$ & 0.641 & $\mathbf{0 . 6 3 3}$ & 0.643 & 0.617 & 0.666 & $\mathbf{0 . 6 7 6}$ & 0.644 \\
PPIEdge & $\mathbf{0 . 6 2 3}$ & $\mathbf{0 . 6 1 8}$ & $\mathbf{0 . 6 5 0}$ & $\mathbf{0 . 6 1 5}$ & $\mathbf{0 . 6 5 8}$ & $\mathbf{0 . 6 6 1}$ & 0.658 & 0.662 & $\mathbf{0 . 6 6 0}$ \\
CNF & $\underline{\mathbf{0 . 7 3 4}}$ & $\underline{\mathbf{0 . 7 2 6}}$ & $\underline{\mathbf{0 . 7 2 7}}$ & $\underline{\mathbf{0 . 7 2 1}}$ & $\mathbf{0 . 7 1 1}$ & $\underline{\mathbf{0 . 7 1 7}}$ & 0.684 & $\underline{\mathbf{0 . 6 9 7}}$ & $\mathbf{0 . 6 5 6}$ \\
\hline
\end{tabular}




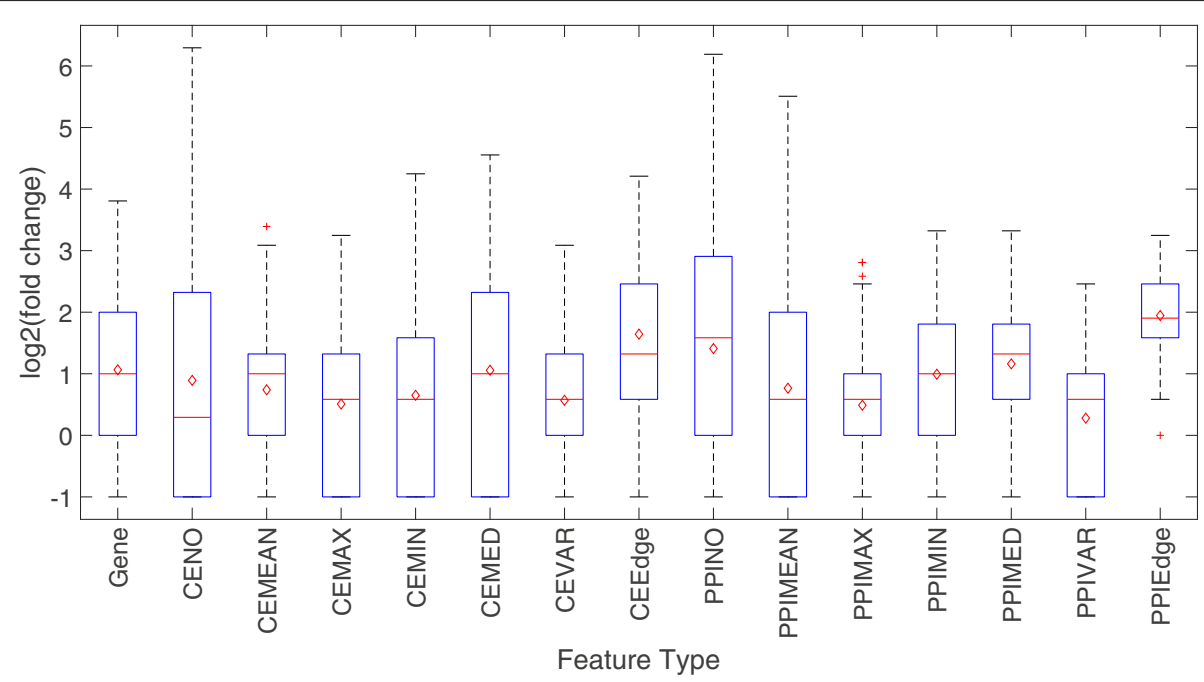

Fig. 6 Feature type stability. Boxplot of the fold change of overlapping gene signatures in pairwise setting across 12 studies. Fold change values were converted to log scale. Red diamond denotes the geometric mean of the fold change values

tion models. This is consistent with the results reported in [21], but in contradiction with the findings in two previous studies, where network-based features were shown to provide no benefit in classification [23, 24]. The discrepancy may be attributed to the fact that we did not perform any feature selection for any of the feature types in our study in contrast to other studies. Supervised feature selection as performed in other studies may introduce overfitting, given a large number of features and a modest number of samples.

As the MEAN, MIN, MAX, and MEDIAN operators on both gene co-expression and protein-protein interaction networks seem to be beneficial but mutually complementary to each other (data not shown), we tested whether the combination of these features can improve prediction performance further. Indeed, by merging the classifiers from different network-based features into an ensemble classifier (CNF), the highest classification AUC was observed on the ACES dataset (Fig. 4), signifying the benefit of combining different network features for improved prediction accuracy.

\section{Classification accuracy on smaller sub-samples of ACES dataset}

To understand why the network-based features worked well in the combined $A C E S$ dataset, but not in many of individual cohorts, the effect of sample sizes on classification accuracy was investigated to determine its relationship with the classification accuracy of network-based features. For this analysis, 10 sub-samples consisting of the same number of patients were created for specific percentages (from 90\% down to 10\%) of the ACES dataset. Then, similar 5 -fold cross-validations were performed for 10 repetitions of each of the 10 sub-samples. The average
AUC scores of each feature type and different sub-sample percentages are shown in Fig. 5. From Fig. 5, it can be observed that while gene-based feature have relatively stable performance with regard to different sample sizes, the network-based features, in particular, MEAN, MAX, MIN, and MEDIAN, from both co-expression and PPI networks outperformed gene-based feature only for relatively larger sample sizes, and can be much worse than gene-based feature for small sample sizes. Even more consistent trend is observed on the ensemble classifier combining different network features: average AUC for the CNF classifier decreases steadily with the decrease of sample sizes. Given that no consistent improvement of network-based features over gene-based feature was found on the 12 individual cohorts (Fig. 3), it can be concluded that a minimum number of samples are needed to obtain an improved and stable prediction accuracy using network-based features. This results aligns well with other studies where datasets were merged together for improved accuracy $[9,10]$.

\section{Robustness of features across patient cohorts}

Figure 6 shows the robustness value for different feature types. Overall, network-based features do not seem to have

Table 3 GO analysis of gene-based feature

\begin{tabular}{llll}
\hline Feature & Term & Count & $\begin{array}{l}\text { Benjamini corrected } \\
p \text {-value }\end{array}$ \\
\hline Gene-based & Cell cycle & 138 & $2.20 \mathrm{E}-15$ \\
& DNA repair & 42 & $1.90 \mathrm{E}-01$ \\
& cell-cell adhesion & 49 & $4.4 . \mathrm{E}-1$ \\
& p53 signaling pathway & 20 & $1.70 \mathrm{E}-02$
\end{tabular}


significantly higher overlap than gene-based feature, contrary to claims made by several recent studies [12, 21, 27], but consistent with results reported in [23]. Three networkbased features, CEEdge, PPIEdge, and PPINO appear to be the most robust among the 15 feature types and the extent of overlap is higher than gene-based features. However, further investigation revealed that the top-ranked CEEdge and PPIEdge features tend to involve genes with higher

Table 4 GO analysis of network-based feature types

\begin{tabular}{|c|c|c|c|c|c|c|}
\hline \multirow{2}{*}{ Feature Type } & \multicolumn{3}{|l|}{ Co-expression Network } & \multicolumn{3}{|l|}{ PPI network } \\
\hline & Term & Count & $\begin{array}{l}\text { Benjamini } \\
\text { corrected } \\
p \text {-value }\end{array}$ & Term & Count & $\begin{array}{l}\text { Benjamini } \\
\text { corrected } \\
p \text {-value }\end{array}$ \\
\hline \multirow[t]{4}{*}{ NO } & Growth factor & 24 & 4.10E-02 & $\begin{array}{l}\text { G-protein coupled receptor } \\
\text { signaling pathway }\end{array}$ & 49 & $9.40 \mathrm{E}-01$ \\
\hline & $\begin{array}{l}\text { Jak-stat signaling } \\
\text { pathway }\end{array}$ & 28 & 1.70E-01 & Jak-stat signaling pathway & 19 & 4.30E-01 \\
\hline & Cell junction & 49 & $5.90 \mathrm{E}-01$ & Cell junction & 42 & $1.80 \mathrm{E}-01$ \\
\hline & Olfactory transduction & 13 & $6.40 \mathrm{E}-01$ & Extracellular region & 111 & $6.60 \mathrm{E}-02$ \\
\hline \multirow[t]{5}{*}{ MEAN } & RNA transport & 33 & 3.30E-01 & Transmembrane & 479 & 1.60E-24 \\
\hline & Lysosome & 40 & 5.00E-01 & Extracellular matrix & 37 & 2.30E-03 \\
\hline & $\begin{array}{l}\text { Antigen processing and } \\
\text { presentation }\end{array}$ & 19 & 3.60E-01 & Bicellular tight junction & 14 & $8.80 \mathrm{E}-01$ \\
\hline & $\begin{array}{l}\text { Endothelial cell } \\
\text { chemotaxis }\end{array}$ & 5 & $9.90 \mathrm{E}-01$ & Notch signaling pathway & 6 & $9.2 \mathrm{E}-1$ \\
\hline & & & & $\begin{array}{l}\text { Leukocyte transendothelial } \\
\text { migration }\end{array}$ & 10 & 9.7E-01 \\
\hline \multirow[t]{5}{*}{ MAXIMUM } & Cell cycle & 132 & $2.40 \mathrm{E}-12$ & Cell cycle & 122 & $2.70 \mathrm{E}-10$ \\
\hline & $\begin{array}{l}\text { Antigen processing and } \\
\text { presentation }\end{array}$ & 27 & 7.70E-03 & $\begin{array}{l}\text { Positive regulation of canonical } \\
\text { Wnt signaling pathway }\end{array}$ & 36 & $1.60 \mathrm{E}-05$ \\
\hline & DNA repair & 45 & $5.20 \mathrm{E}-02$ & $\begin{array}{l}\text { T-Cell receptor signaling } \\
\text { pathway }\end{array}$ & 51 & $1.20 \mathrm{E}-09$ \\
\hline & $\begin{array}{l}\text { Cardiac epithelial to } \\
\text { mesenchymal transition }\end{array}$ & 5 & $9.9 \mathrm{E}-01$ & Cell-cell adherens junction & 70 & $3.50 \mathrm{E}-05$ \\
\hline & & & & $\begin{array}{l}\text { Positive regulation of blood } \\
\text { vessel endothelial cell } \\
\text { migration }\end{array}$ & 7 & 5.7E-01 \\
\hline \multirow[t]{3}{*}{ MINIMUM } & Cell cycle & 116 & 2.00E-06 & Cell cycle & 136 & $5.90 \mathrm{E}-14$ \\
\hline & p53 signaling pathway & 5 & $9.00 \mathrm{E}-01$ & p53 signaling pathway & 19 & 4.90E-02 \\
\hline & $\begin{array}{l}\text { Rho cell motility signaling } \\
\text { pathway }\end{array}$ & 7 & $9.60 \mathrm{E}-01$ & DNA repair & 41 & $2.80 \mathrm{E}-01$ \\
\hline \multirow[t]{3}{*}{ MEDIAN } & Cell cycle & 124 & 2.80E-09 & Sensory transduction & 26 & $9.90 \mathrm{E}-01$ \\
\hline & DNA repair & 47 & 1.70E-02 & Telomere & 4 & $9.80 \mathrm{E}-01$ \\
\hline & $\begin{array}{l}\text { CSonic Hedgehog }(\mathrm{SHH}) \\
\text { Receptor Ptc1 Regulates } \\
\text { cell cycle }\end{array}$ & 3 & $9.90 \mathrm{E}-01$ & Ribosome & 17 & $9.80 \mathrm{E}-01$ \\
\hline \multirow[t]{3}{*}{ VARIANCE } & Cell cycle & 138 & 6.30E-18 & $\begin{array}{l}\text { Intracellular steroid hormone } \\
\text { receptor signaling pathway }\end{array}$ & 7 & $9.40 \mathrm{E}-01$ \\
\hline & DNA repair & 47 & 8.80E-04 & Extracellular space & 177 & 2.10E-01 \\
\hline & $\begin{array}{l}\text { Positive regulation of } \\
\text { telomere maintainance }\end{array}$ & 12 & $8.00 \mathrm{E}-02$ & Immune response & 64 & $9.50 \mathrm{E}-01$ \\
\hline \multirow[t]{4}{*}{ EDGE } & Cell cycle & 147 & $1.80 \mathrm{E}-22$ & Cell cycle & 167 & 4.80E-30 \\
\hline & $\begin{array}{l}\text { Positive regulation of } \\
\text { telomere maintainance }\end{array}$ & 15 & 1.30E-03 & Cell-cell adherens junction & 84 & $5.00 \mathrm{E}-10$ \\
\hline & DNA repair & 44 & 7.20E-03 & $\begin{array}{l}\text { Positive regulation of epithelial } \\
\text { to mesenchymal transition }\end{array}$ & 7 & $9.5 \mathrm{E}-01$ \\
\hline & Cell-cell adhesion & 48 & 1.30E-01 & Regulation of cell motility & 6 & $9.8 \mathrm{E}-01$ \\
\hline
\end{tabular}


degree (mean degree equal to 43 and 102, compared to the network average of 25 and 28, for co-expression network and PPI network, respectively). Therefore, the improved robustness may simply reflect a selection bias as there are few hub nodes in each network, which increases the chance of feature overlap between different datasets. Similarly, the improved robustness of PPINO was due to an inherent bias in including low-degree genes, where the median degree of the top-160 features was zero in 6 out of the 12 studies. We suspect that this type of biases is the main reason for the high robustness of network-based features claimed in the literature and a more comprehensive re-evaluation of the previously reported results may be deserved.

\section{Gene ontology (GO) analysis of the feature types}

To investigate the biological function of the top-ranked features, we combined the top-160 genes for each feature type from all 12 cohorts and performed gene ontology enrichment analysis using the combined gene list. Tables 3 and 4 show top-ranked GO terms along with FDR-corrected $p$-values for gene- and network-based features respectively. Overall, cell cycle and DNA damage are the most recurring GO terms, appearing in most feature types. Other well-known metastasis-related terms such as cell-cell adhesion, cell junction, and various signaling pathways including p53 and Jak-stat appear in several feature types, but the statistical significance of the enrichment is rather low, partly due to the FDR correction required to tackle with the multiple hypothesis testing problem. This is in agreement with the low robustness of the features across datasets, and suggests that a combination of diverse features from large cohorts as in this study is necessary for both mechanistic understanding and improved prediction of metastatic breast cancer.

\section{Conclusions}

Improved prediction accuracy and signature stability across multiple datasets are very crucial for the prediction and mechanistic understanding of breast cancer metastasis. Here we present a comprehensive analysis of distinct network-based features in comparison to gene-based feature. In general, the number of patients and the ratio between metastatic and non-metastatic patients in the dataset can dramatically impact the number of significant features that can be detected, for both gene-based and network-based features. While network-based features can provide higher prediction accuracy than genebased features in large cohorts, its performance gain diminishes in smaller dataset. We did not find strong evidence to support the claim that network-based features are more stable than gene-based feature (in fact, some potential bias that could have lead to the false claim was identified). In addition, gene ontology analysis revealed relatively insignificant enrichment of known metastasisrelated pathways. Nevertheless, an ensemble classifier combining different network features achieved significantly higher accuracy than gene-based and individual network-based features, signifying both the potential and challenges in network-based prediction and understanding of breast cancer metastasis.

\section{Acknowledgements \\ We thank the anonymous reviewers for their constructive comments which have helped improve the manuscript.}

\section{About this supplement}

This article has been published as part of BMC Medical Genomics Volume 13 Supplement 5, 2020: The International Conference on Intelligent Biology and Medicine (ICIBM) 2019: Computational methods and application in medical genomics (part 1). The full contents of the supplement are available online at https://bmcmedgenomics.biomedcentral.com/articles/supplements/volume13-supplement-5.

\section{Authors' contributions}

$J R$ and NA conceived of the study and designed the experiments. NA performed the experiments, data analysis, and drafted the manuscript under the supervision of JR. ZL and TH provided biological background of breast cancer metastasis and helped with biological interpretation of the results. All authors read and approved the final manuscript.

Funding

This research and this article's publication costs were supported by NSF grant ABI-1565076 and NIH grant U54CA217297.

Availability of data and materials

The datasets used and/or analysed during the current study are available from the corresponding research article.

Ethics approval and consent to participate

Not applicable.

Consent for publication

Not applicable.

Competing interests

The authors declare that they have no competing interests.

Published: 3 April 2020

References

1. Weigelt $B$, Peterse $J$, van't Veer $L$. Breast cancer metastasis: markers and models. Nat Rev Cancer. 2005;5(8):591-602.

2. Siegel RL, Miller KD, Jemal A. Cancer statistics, 2018. CA: Cancer J Clin. 2018;68(1):7-30.

3. Breast Cancer - Metastatic: Statistics. https://www.cancer.net/cancertypes/breast-cancer-metastatic/statistics. Accessed 20 Feb 2019.

4. McGuire WL. Breast Cancer Prognostic Factors: Evaluation Guidelines. JNCl: J Natl Cancer Inst. 1991;83(3):154-5.

5. van 't Veer $L$, Dai H, van de Vijver MJ, He YD, Hart AAM, Mao M, Peterse HL, van der Kooy K, Marton MJ, Witteveen AT, Schreiber GJ, Kerkhoven RM, Roberts C, Linsley PS, Bernards R, Friend SH. Gene expression profiling predicts clinical outcome of breast cancer. Nature. 2002;415:530

6. van de Vijver MJ, He YD, van 't Veer LJ, Dai H, Hart AAM, Voskuil DW, Schreiber GJ, Peterse JL, Roberts C, Marton MJ, Parrish M, Atsma D, Witteveen A, Glas A, Delahaye L, van der Velde T, Bartelink $H_{\text {, }}$ Rodenhuis S, Rutgers ET, Friend SH, Bernards R. A gene-expression signature as a predictor of survival in breast cancer. N Engl J Med. 2002;347(25):1999-2009.

7. Wang Y, Klijn JG, Zhang Y, Sieuwerts AM, Look MP, Yang F, Talantov D, Timmermans M, Gelder MEM- $v$, Yu J, Jatkoe T, Berns EM, Atkins D, Foekens JA. Gene-expression profiles to predict distant metastasis of lymph-node-negative primary breast cancer. Lancet. 2005;365(9460): $671-9$. 
8. Givol D, Domany E, Getz G, Kela I, Ein-Dor L. Outcome signature genes in breast cancer: is there a unique set? Bioinformatics. 2004;21(2):171-8.

9. van Vliet MH, Reyal F, Horlings HM, van de Vijver MJ, Reinders MJ, Wessels LF. Pooling breast cancer datasets has a synergetic effect on classification performance and improves signature stability. BMC Genomics. 2008;9:375.

10. Shen R, Ghosh D, Chinnaiyan AM. Prognostic meta-signature of breast cancer developed by two-stage mixture modeling of microarray data. BMC Genomics. 2004;5:94.

11. Chou H-L, Yao C-T, Su S-L, Lee C-Y, Hu K-Y, Terng H-J, Shih Y-W, Chang Y-T, Lu Y-F, Chang C-W, Wahlqvist ML, Wetter T, Chu C-M. Gene expression profiling of breast cancer survivability by pooled cdna microarray analysis using logistic regression, artificial neural networks and decision trees. BMC Bioinformatics. 2013;14(1):100.

12. Chuang H-Y, Lee E, Liu Y-T, Lee D, Ideker T. Network-based classification of breast cancer metastasis. Mol Syst Biol. 2007;3(1):. https://doi.org/10. 1038/msb4100180.

13. Park MY, Hastie T, Tibshirani R. Averaged gene expressions for regression. Biostatistics. 2006;8(2):212-27.

14. Pujana MA, Han J-DJ, Starita LM, Stevens KN, Tewari M, Ahn JS, Rennert G, Moreno V, Kirchhoff T, Gold B, Assmann V, ElShamy WM, Rual J-F, Levine D, Rozek LS, Gelman RS, Gunsalus KC, Greenberg RA, Sobhian B, Bertin N, Venkatesan K, Ayivi-Guedehoussou N, Solé X, Hernández P, Lázaro C, Nathanson KL, Weber BL, Cusick ME, Hill DE, Offit K, Livingston DM, Gruber SB, Parvin JD, Vidal M. Network modeling links breast cancer susceptibility and centrosome dysfunction. Nat Genet. 2007;39:1338.

15. Lee $\mathrm{E}$, Chuang H-Y, Kim J-W, Ideker T, Lee D. Inferring Pathway Activity toward Precise Disease Classification. PLoS Comput Biol. 2008;4(11): https://doi.org/10.1371/journal.pcbi.1000217.

16. Taylor IW, Linding R, Warde-Farley D, Liu Y, Pesquita C, Faria D, Bull S, Pawson T, Morris Q, Wrana JL. Dynamic modularity in protein interaction networks predicts breast cancer outcome. Nat Biotechnol. 2009;27(2): 199-204.

17. Schönhuth A, Davicioni E, Moser F, Ester M, Dao P, Salari R, Colak R. Inferring cancer subnetwork markers using density-constrained biclustering. Bioinformatics. 2010;26(18):625-31.

18. Abraham G, Kowalczyk A, Loi S, Haviv I, Zobel J. Prediction of breast cancer prognosis using gene set statistics provides signature stability and biological context. BMC Bioinformatics. 2010;11(1):277.

19. Akker Evd, Verbruggen B, Heijmans B, Beekman M, Kok J, Slagboom E, Reinders M. Integrating Protein-Protein Interaction Networks with GeneGene Co-Expression Networks improves Gene Signatures for Classifying Breast Cancer Metastasis. J Integr Bioinforma. 2011;8(2):222-38.

20. Winter C, Kristiansen G, Kersting S, Roy J, Aust D, Knösel T, Rümmele P, Jahnke B, Hentrich V, Rückert F, Niedergethmann M, Weichert W, Bahra M, Schlitt HJ, Settmacher U, Friess H, Büchler M, Saeger H-D, Schroeder M, Pilarsky C, Grützmann R. Google Goes Cancer: Improving Outcome Prediction for Cancer Patients by Network-Based Ranking of Marker Genes. PLoS Comput Biol. 2012;8(5):. https://doi.org/10.1371/journal.pcbi. 1002511.

21. Allahyar A, de Ridder J. FERAL: network-based classifier with application to breast cancer outcome prediction. Bioinformatics. 2015;31(12):311-9.

22. Alcaraz N, Vandin F, Baumbach J, Ditzel HJ, List M, Batra R. De novo pathway-based biomarker identification. Nucleic Acids Res. 2017;45(16): 151.

23. Staiger C, Cadot S, Kooter R, Dittrich M, Müller T, Klau GW, Wessels LFA. A Critical Evaluation of Network and Pathway-Based Classifiers for Outcome Prediction in Breast Cancer. PLoS ONE. 2012;7(4):. https://doi. org/10.1371/journal.pone.0034796.

24. Staiger C, Cadot S, Györffy B, Wessels L, Klau G. Current compositefeature classification methods do not outperform simple single-genes classifiers in breast cancer prognosis. Front Genet. 2013;4:289.

25. BIOGRID. https://downloads.thebiogrid.org/BioGRID/Release-Archive/ BIOGRID-3.4.149/. Accessed 10 Dec 2018.

26. Benjamini $Y$, Hochberg $Y$. Controlling the false discovery rate: A practical and powerful approach to multiple testing. J R Stat Soc Ser B (Methodol). 1995;57(1):289-300

27. Jahid MJ, Ruan J. A steiner tree-based method for biomarker discovery and classification in breast cancer metastasis. BMC Genomics. 2012;13:. https://doi.org/10.1186/1471-2164-13-s6-s8.

\section{Publisher's Note}

Springer Nature remains neutral with regard to jurisdictional claims in published maps and institutional affiliations.
Ready to submit your research? Choose BMC and benefit from:

- fast, convenient online submission

- thorough peer review by experienced researchers in your field

- rapid publication on acceptance

- support for research data, including large and complex data types

- gold Open Access which fosters wider collaboration and increased citations

- maximum visibility for your research: over 100M website views per year

At $\mathrm{BMC}$, research is always in progress.

Learn more biomedcentral.com/submissions 